\title{
Berichtigung
}

\section{Röntgeninterferenzen an kolloiden Systemen}

Von R. Hosemann (Treysa)

Kolloid-Zeitschrift 117, 13 (1950)

S. 18: Statt Gl. (32) schreibe 199) und statt (99) eine Zeile tiefer nun (32). - - In Gl. (24) ist der Faktor $-(n+3,5)$ zu streichen.

S. 20: In Gl. (24) ist der Faktor $-(n+3,5)$ zu streichen. - In Tabelle 1 lautet die letzte Zahl in der Zeile für die Größe $S(n)$ 2,83 statt 2,38. - In Abb. 5 ist Näherung (15) durch - - - dargestellt.

S. 23: In Gl. (47) muß $w>1$ statt $w<1$ stehen.

S. 25: Gl. (61) muß lauten

$$
G(x)=\left\{\begin{array}{l}
1 \text { für }|x| \leqq z=y+z \\
0 \text { für }|x|>z .
\end{array}\right.
$$

Gl. (63) muß lauten

$$
A_{4}=Q_{4} \cdot 3 \frac{\sin u z-u z \cos u z}{(u z)^{3}} .
$$

Die letzte Gleichung unten rechts gilt für $G(0)$.

S. 27: Abb. 10 bezieht sich auf Gl. (82) [nicht (83)]. S. 30: Gl. (103) muß lauten

$$
J_{1}=C_{3} \frac{1}{\left(1+k^{2} \varepsilon^{2}\right)^{\frac{n+4}{2}}} .
$$

S. 33: Der Nenner von (115) lautet $\left|\varphi_{1 \max }\right| \cdot-$ Der Nenner von (116) ist ins Quadrat zu erheben.

S. 34: Setze in Gl. (125) und (126) statt $\sqrt{10}$ nun $\sqrt{5}$ S. 35: In der 15. Zeile von rechts oben steht statt $J_{3}^{\prime}$ richtig $\mathrm{J}_{3}^{\prime \prime}$.

S. 40: Fünf Zeilen über der Zusammenfassung ist statt $Z$ itat ${ }^{29}$ ) nun ${ }^{19}$ ) zu setzen.

\section{Bücherbesprechungen}

Leçons sur les fluides visqueux (Vorlesungen über zähe Flüssigkeiten). Von $\mathrm{H}$. Vill at-Paris. Gesam. u. herausg. von J. Kravtch en ko-Paris. VIII, 540 S. u. $17 \mathrm{Abb}$. (Paris 1943, GauthierVillars, Impr. Edit.). Preis brosch. ffr. 1200,-

Im vorliegenden Band handelt es sich nach den eigenen Worten des Verfassers nicht um ein systematisches Lehrbuch der Hydrodynamik zäher Flüssigkeiten, vielmehr um eine Einführung in eine solche vollständigere Darstellung. Den Unterschieà zwischen beiden sieht der Verfasser darin, daß nur eine Auswahl besonders wichtiger Probleme. diese allerdings mit großer Gründlichkeit, behandelt wird.

Wer den Verfasser als Autor wesentlicher mathematischer Beiträge zur Hydrodynamik kennt, wird sich nicht wundern, wenn die vorliegende „Einführung“ von einem hohen Standpunkt aus erfolgt, die der mathematischen Strenge aufs ÄuBerste Rechnung trägt. Dabei beschränkt sie sich. durchatis nicht auf eine strenge Darlegung derGrundlagen, sondern führt in den ausgewählten Problemen bis in die neueren und neuesten Ergebnisse so im ersten Teil, der den $\mathrm{Navier}$ schen Gleichungen gewidmet ist, bis zu dem Odquistschen Tensor, und im zweiten, der die exakten Lösungen der $\mathrm{Navieirschen} \mathrm{Gleichungen} \mathrm{behandelt,}$ über Oseen-Faxén bis zu einer Verallgemeinerung der Pois e uill eschen Bewegung nach neueren Untersuchungen von $S z y$ mans 1 i. Der dritte Teil ist der Stabilität der Bewegung zäher Flüssigkeiten nach neueren Arbeiten von $S$ ynge u. a. mit Berichtigung von Ra yleigh schen Folgerungen - gewidmet.

Alles in allem: beste strenge. Theorie, und nur dies, im Gegensatz etwa zu dem mehr, ,experimentellen" Brillouin (Leçons sur la viscosité des liquides et des gaz). ",Re ynoldssche Zahl" nur in einer Fußnote, "Grenzschicht" tritt garnicht auf. Trotzdem: in seiner Art ein ausgezeichnetes Werk!

$$
\text { L. Schiller (Weilburg/L.) }
$$

Praktikum der quantitativen anorg. Analyse, Von H. L ux-München 2. Aufl. VIII, 183 S., 247 Abb. (München 1949, Verlag J. F. Bergmann). Preis DM 8,-.

Das Erscheinen dieser Neuauflage des ursprünglich aus dem bekannten Praktikum von A. S tock und A.Stähler hervorgegangenen Buches ist sehr zu begrüßen. - An der Neuauflage sind besonders hervorzuheben die sehr geschickt angeordneten und kurz gefaBten allgemeinen praktischen Anweisungen und die Abschnitte „Allgemeines", die den einzelnen Kapiteln vorangehen. Die anschließenden Vorschriften sind auch für den Anfänger gut verständlich dargestellt. An jede Vorschrift anschließende Erläuterungen sind besonders wertvoll. Das Buch, das gewichtsanalytische Einzelbestimmungen, maßanalytische und elektroanalytische Methoden ausführlich behandelt, beschäftigt sich am Schluß in gedrängter Kürze auch mit Trennungen und gibt eine Anzahl von Beispielen für die Analyse von Mineralien und technischen Produkten. Es ist zur Verwendung itn Anfängerpraktikum sehr zu empfehlen. Holluta (Karlsiuhe)

Lehrbuch der organischen Chemie. Von W. L a ng e n beck-Rostock, 8. Auflage, XVI, $541 \mathrm{~S}$., mit $5 \mathrm{Abb}$. (Dresden und Leipzig 1949, Verlag von Theodor Steinkopff). Preis geb. DM 15,-.

Das bekannte Lehrbuch der organischen Chemie erscheint nunmehr in der 8. Aufl. An seiner besonderen, glücklichen Einteilung hat sich nichts geändert. In einem ,,ersten Buch" werden die einfachen organischen Verbindungen, unterteilt in aliphatische Verbindungen, aromatische Verbindungen, heterocyklische Verbindungen, erörtert. Der Stoff entspricht nach Umfang und Anordnung dem, was als Ergänzung für die Experimentalvorlesung in organischer Chemie für Anfänger aller Studienrich. tungen (Chemiker, Physiker, Naturwissenschaftler, Techniker, Mediziner) wichtig ist.

Das „zweite Buch" gibt gegenüber den Lehrbüchern mit ähnlichen Stoffen den besonderen Charakter. Es behandelt spezielle Arbeitsgebiete: Koh- 